\title{
A Hybrid Technique for TCAD Modeling and Optimization
}

\author{
B. Govoreanu ${ }^{a, b}$, J. Kopalides ${ }^{b}$, W. Schocnmaker ${ }^{b}$, G. Dima ${ }^{a}$, \\ O. Mitrea ${ }^{a}$, M.D. Profirescu ${ }^{a}$ \\ ${ }^{a}$ Technical University of Bucharest, Blvd. I. Maniu 1-3, Bucharest 6, Romania \\ ${ }^{6}$ IMEC, ASP Division, Kapeldreef 75, B-3001 Leuven, Belgium
}

\begin{abstract}
This paper focuses on continuous simulated annealing global optimization method to be used in conjunction with statistical response surface modeling and powerful local optimization techniques to improve the design and analysis using TCAD.
\end{abstract}

\section{Introduction}

A continuously evolving area of TCAD applications is the modeling and optimization of IC manufacturing processes. Many steps have been done towards automating computer design of experiments (DOE), response surface modeling (RSM) and optimization. Integrated software packages offer solutions dealing with such tasks. RSM provides an efficient method to obtain a coarse grain picture of the behaviour of the system under consideration. Since computer experiments are deterministic, it makes sense to build models which pass through the experimental points. This is done by the mean of statistical RSM (sRSM) where the interpolation is realized either by considering the experiments as a particular realization of a stochastic process, [1] (parametric sRSM) or by using Feynman path integrals, [2] (non-parametric sRSM). This is a useful approach provided the system under experimentation has a strong non-linearity and there is a lack of prior knowledge about the shapes of its response. However, optimization of such sRSM becomes a difficult task for high-dimensional problems and the traditional optimization algorithms can fail. In order to avoid this problem, we have implemented a probabilistic global optimization method which collects global information characterising the coarse grain shapes of the system, i.e. locating good regions for further analysis. It can be used in an iterative way to succesively analyze and zoom in the region under exploration up to the point a local optimization method can be succesfully applied.

\section{Simulated Annealing}

The simulated annealing-like techniques (SA) provide a powerful class of global optimization Monte Carlo methods which offer statistical guaranties to find an optimal solution for multivariate, multimodal objective functions whose characteristics include 
strong non-linearities, discontinuities and stochasticity [3]. It does not involve objective gradient evaluations (unless the objective itself is a sensitivity based function) and can process complex constraints. However, it requires many objective evaluations, being time consuming. Our work focused on the implementation of an adaptive SA to perform the optimization problem over a continuous $D \in R^{n}$ hyper-rectangular box with bound constraints applied to both input and output variables.

Basically, to solve an optimization problem with a SA method, one must be able to define the following elements: (a) a configuration space $(D)$ and a set of states, $\vec{x}$, belonging to this space; (b) a rule for updating the current state in configuration space; (c) a cost function assigned to each state; (d) an adaptive updating mechanism for some control parameters of the algorithm, i.e. a cooling schedule. The main control parameter of the algorithm is the "temperature", $(T)$ based on an analogy with the physical process of annealing and the cost function is the "energy" of the system. Thus, the SA algorithm can be seen as an evolution towards the ground state of the minimum energy of an annealed system whose temperature is gradually reduced so that at each value of $T$, the system reaches quasi-equilibrium condition. Under some less restrictive assumptions, the evolution of the average cost $E$ and standard deviation of the cost $\sigma$ is given by:

$$
E(T)=\left\{\begin{array}{ll}
E_{\text {init }}-\frac{\sigma_{\text {init }}^{2}}{T} & T>T_{l} \\
E_{\text {init }}-\frac{\sigma_{\text {init }}^{2}}{T_{l}}\left(2-\frac{T}{T_{l}}\right) & T<T_{l}
\end{array} \quad \sigma(T)= \begin{cases}\sigma_{\text {init }} & T>T_{l} \\
\sigma_{\text {init }} \frac{T}{T} & T<T_{l}\end{cases}\right.
$$

where the index init denotes initial values and $T_{l}$ is the transistion temperature between weak and strong control regions. in the temperature range. SA spends most of its time in the strong control region. The temperature can be updated at each costfunction evaluation, being an inhomogenous approach or it may be kept at a fixed value during a series of cost-function evaluations, which is a homogenous method, i.e. quasi-equilibrium is required for temperature updating.

Our approach is a combination between these two extreme approaches. We choose a finite-range state generation mechanism which allows an exponential decrease of the control parameter, but we perform its update after a number of objective evaluations which provides an estimate of the amount which has to be subtracted from $T$, based on particular statistic properties of the cost function. Random numbers $r_{i} \in[-1,1]$ are generated with the probability density function:

$$
f\left(r_{i}\right)=\frac{1}{2 \ln \left(1+\frac{1}{K_{i}}\right)\left(\left|r_{i}\right|+K_{i}\right)}
$$

where $K_{i}$ are some adaptive parameters depending on the instance at hand. Then input parameters are updated based on $x_{i+1}=x_{i}+r_{i}\left(H_{i}-L_{i}\right)$ under the constraint $x_{i+1} \in\left[L_{i}, H_{i}\right]$, where $H_{i}$ and $L_{i}$ define the search box.

Unlike traditional implementations [4], to find a good approximation of the quasiequilibrium condition, our approach is based on the fact that if a state can be sampled infinitely often in time (IOT) with probability (2), then an exponential decrease of the temperature still guarantees to find the global optimum. Moreover, we do not use an inhomogenous algorithm as in [3], in order to avoid settings of parameters which are problem dependent and difficult to set by the user. We perform a number of generations at a certain $T_{k}$ and its update is given by: $T_{k+1}=T_{k}-\gamma \frac{T_{k}^{2}}{\sigma\left(T_{k}\right)}$ where $\gamma$ is a factor which results after imposing some conditions to prevent us from a too large updates and $\sigma\left(T_{k}\right)$ is the standard deviation of the cost function at the current temperature. Combining this formula with (1) yields an exponential reduction of the temperature. 


\section{Application Example}

We have applied this optimization technique for process calibration of a $0.5 \mu \mathrm{m}$ Boron implant. Two key parameters which control the implant damage model of Hobler and Selberherr, have been identified as being the most sensitive ones. These are D.PLUS to determine the number of interstitials contributed by the "plus-one" model and D.SCALE to control the number of Frenkel pairs included as damage. The simulations have been driven using IMEC's TCAD system NORMAN/DEBORA and TSUPREM4. The cost function is a weighted squared sum of eleven sRSM error functions (see Fig.1). Each error function has the following form:

$$
\operatorname{Err}_{(j)}=\sum\left[1 / N_{e x t r} \sum_{i=1}^{N_{e x t r}}\left(y_{\text {extr }}^{i}-y_{r e f}^{i}\right)^{2}\right]^{1 / 2}
$$

where the sum is taken over $N_{\text {extr }}$ extraction points of a portion of the profile. The values $y_{\text {ref }}^{i}$ and $y_{\text {extr }}^{i}$ correspond to the experimental and simulated profiles for several Boron implants at $15[\mathrm{keV}]$, with a dose of $1 \mathrm{e} 14,5 \mathrm{e} 14,2 \mathrm{e} 15,5 \mathrm{e} 15\left[\mathrm{~cm}^{-2}\right]$. The considered profiles have been splitted in two or more parts to be able to consider higher weights in the more critical regions.

We denote a cycle as being a suite of DOE simulations, followed by SRSM modeling and SA optimization. After each cycle, we select a smaller region which is susceptible to contain the true optimum and continue with another cycle. At the end of a SA optimization we have a large set of states of the system to be optimized available for postprocessing. These can be used to build the probability density function (pdf) of good states which might be interpreted as a negative of the objective function, but with some more properties. It is known that the radial basis functions (RBF) have the property of being universal approximators, [5] and so, these can be used to estimate the pdf of states (see Fig.2). However, the problem of locating a good region is a very difficult task in a high dimensional space, given a $R B F$ based pdf so we have implemented a simpler algorithm which analyses each dimension of the input space separately. The selection is made by extracting the region of the initial search interval, centered on the last point obtained during optimization, which contains a certain percent of the corresponding coordinate of the states visited during a SA optimization. In our implementation, each state was considered to be equally important and thus we did not impose a high percent (e.g. 66\%), but more sophisticated algorithms might be conceived by learning, i.e. by exploiting the fact that the states collected at the end of the optimization are more relevant than those collected at the beginning.

In this example, two cycles were performed. The analysis space has been reduced approximately 3300 times in volume over the two cycles, which is sufficiently small to start a gradient based optimizer DOE over the true system to find the optimal settings. Finally, we got the values 0 and $1.86 \mathrm{e}-05$ for D.PLUS and D.SCALE respectively, which best fit the simulated profiles to the experimental ones, with respect to the error function as defined in (3).

\section{Conclusions}

We systematically applied a model refinement technique driving from global to local models by using a simulated annealing optimization method, to explore a highly nonlinear system in order to locate good regions for further experimentation with the true system instead of its model. In the first stage we analyse models to save CPU times, whereas in the final stage we use the real system to achieve the desired accuracy. 


\section{Acknowledgement}

The authors are acknowledged to the Ministry of the Flemish Community and the Romanian Ministry for Research and Technology for their financial support.

\section{References}

[1] J. Sacks et al "Design of Computer Experiments" Statistical Science, vol. 4, pp. 409-435, 1996

[2] W. Schoenmaker, R. Cartuyvels "Theory and Implementation of a New Interpolation Method Based on Random Sampling" IEEE J. of TCAD 1997 URL address: http://tcad.stanford.edu/tcad-journal/archive/

[3] L. Ingber "Simulated Annealing: Practice versus Theory" J. Mathl. Comput. Modeling, vol. 18, pp. 29-57, 1993

[4] E.H.L. Aarts, P.M.J. van Laarhoven "Simulated Annealing: Theory and Applica-
tions" Kluwer Ac. Publ., 1987

[5] T. Poggio, F. Girossi "Networks for Approximation and Learning" Proceedings of
IEEE, 1990, vol. 78, no. 9, pp.1481-1490
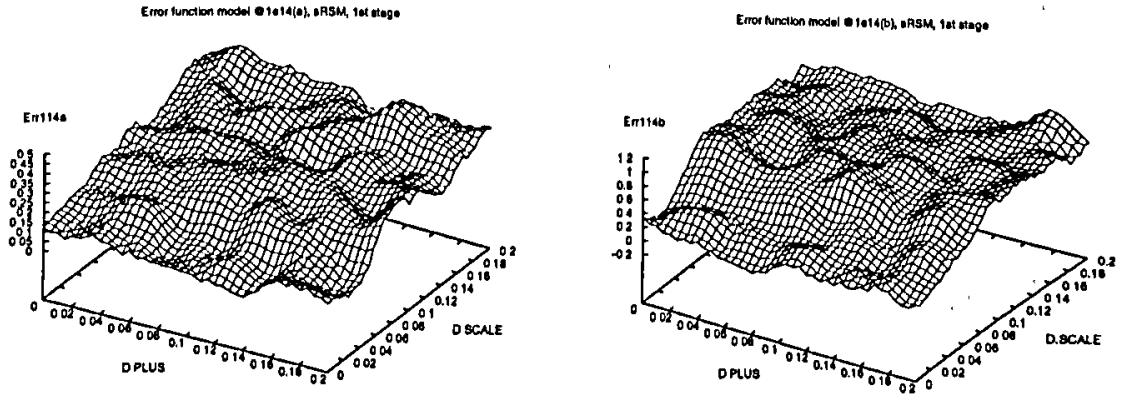

Figure 1: Error function model for a $1 \mathrm{e} 14 \mathrm{~cm}^{-2} \mathrm{~B} 15 \mathrm{keV}$ with non-parametric sRSM
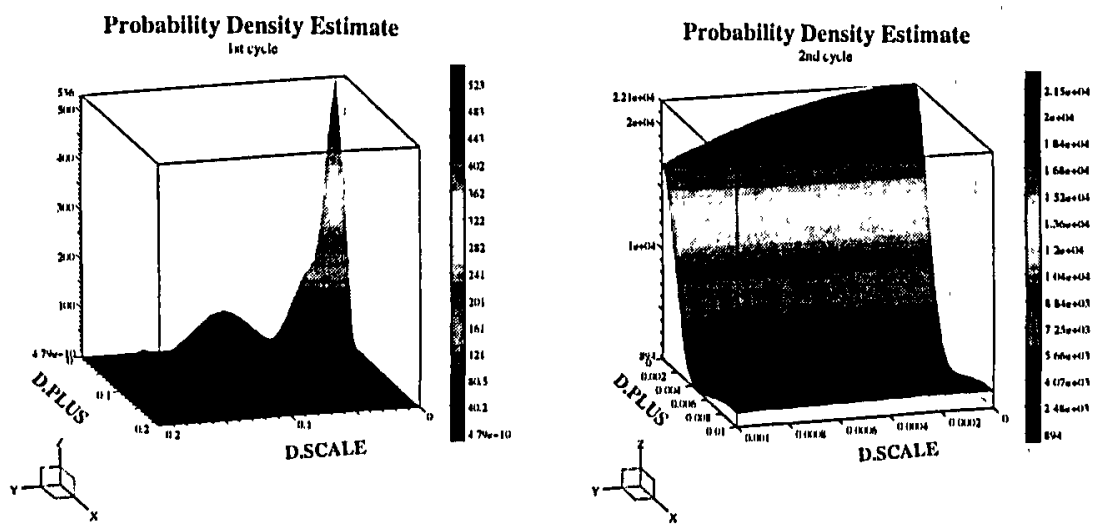

Figure 2: PDF estimates at the end of each modeling-optimization cycle 